\title{
Including side dishes to traditional main meals alter the glycaemic index
}

\author{
S Pirasath ${ }^{1}, \mathrm{~K}$ Thayananthan $^{2}, \mathrm{~S}$ Balakumar $^{3}$, V Arasaratnam $^{4}$ \\ Sri Lanka Journal of Diabetes, Endocrinology and Metabolism 2013; 3: 12-18
}

\begin{abstract}
Objective: To determine the glycaemic index (GI) values of commonly consumed 'idly' based meals in Sri Lanka.

Method: 20 Healthy volunteers (11 males and 9 females) mean ( \pm SD) age and body mass index were $20.10( \pm 0.72)$ years and $21.22( \pm 3.49) \mathrm{kgm}^{-2}$ respectively were selected with their informed written consent. After overnight fasting $75 \mathrm{~g}$ glucose and each test food containing $75 \mathrm{~g}$ digestible carbohydrate were administered on separate days and blood glucose levels measured half hourly for two hours following ingestion. The GI values were calculated and analyzed by Randomized Complete Block Design using SAS analytical package.
\end{abstract}

Results: The mean GI values of 'idly' either with 'sambol' or 'sambol' and plantain ('itharai') or 'sampar' or 'sampar' and plantain ('itharai') or 'sambol' and 'sampar' or 'sambol', 'sampar' and plantain ('itharai') were 56.85 ( \pm 6.26$), 51.10( \pm 6.57), 70.32( \pm 8.22), 67.45( \pm 7.87), 63.99( \pm 3.29)$ and $61.30( \pm 3.09) \%$ respectively.

\begin{abstract}
Interpretation: Based on these GI values, 'idly' with 'sambol' and plantain ('itharai') is of lower GI value (<55\%). 'Idly' either with 'sambol' or 'sampar' and plantain ('itharai') or 'sambol' and 'sampar' or 'sambol', 'sampar' and plantain ('itharai') are of medium GI value (55 to 70\%). 'Idly' with 'sampar' alone is high $\mathrm{GI}$ value (>70 \%). When plantain ('itharai') was given with 'idly', the GI values were decreased, while mixed with 'sampar', the GI values were increased. The GI values were increased when the foods were consumed with 'sampar' alone or 'sampar' and 'sambol'. Thus, when consuming basic traditional meals mixing with foods with different side dishes significantly alters the GI value.
\end{abstract}

Conclusion: Therefore, when dietary advice is given to diabetics and those at risk of coronary heart disease, the side dishes to the basic foods must be considered. The lower GI value foods are better choices for these patients.

Key words: Glycaemic index, glycaemic response, idly, fruits

\section{Introduction}

The glycaemic index (GI) is an important parameter which compares the hyperglycaemic effect of a tested meal with that of a standard dietary carbohydrate dose (1). The blood glucose response to a particular food is reflected by its glycaemic index defined as the incremental area under the blood glucose response curve elicited over a two-hour period following a $75 \mathrm{~g}$ carbohydrate portion of the food, expressed as a percentage of the response to the same amount of carbohydrate from a standard food taken by the same subject $(2,3)$. Foods with GI values of 70 or more are considered to be high GI diet, an index value between 55 to 69 as medium GI diet and less than 55 as low GI diets (4).
Reducing the GI of carbohydrate rich foods in diet would decrease the metabolic risk $(5,6)$. A reduction and stabilization of blood glucose level can reduce the insulin demand and have beneficiary effects on insulin sensitivity, lipid profiles $(7,8)$ and $\beta$-cell functions $(9)$. Reductions in hyperglycemia and hyperinsulinemia may also lower oxidative stress (10) which exacerbates a number of features of the metabolic syndromes (11) including obesity (12) insulin resistance $(13,14,15)$ inflammation and hypertension (16). Prolonged exposure to hyperglycemia can promote macro- (17) and micro-vascular diseases $(18,19)$. Dietary glycaemic index is inversely associated with total Moos Menstrual Distress questionnaire (MDQ) score in the premenstrual phase (20) and Parkinson's disease (21). High glycaemic index is also associated with liver steatosis (22).

${ }^{1}$ Demonstrator, ${ }^{2}$ Technical Officer, ${ }^{3}$ Senior Lecturer, Department of Biochemistry, Faculty of Medicine, University of Jaffna, ${ }^{4}$ Senior Professor in Biochemistry, Vice-Chancellor, University of Jaffna, Sri Lanka. 
The aim of this study was to determine the GI values of commonly consumed idly mixed meals to recommend to patients with diabetes, obesity and cardio vascular disease. The evaluation of GI will help the local public to decide on the foods which have to be consumed. This study focused on the GI values of 'idly' mixed in various traditional combinations of 'sambol' 'sampar' and plantain.

\section{Methods and materials}

\section{Materials}

The pure glucose (Royal Pure Glucose, Smithkline Beecham Pvt Ltd, Moratuwa), 'idly', 'sambol', sambar and plantain ('itharai') were used for this study.

\section{Preparations of foods}

'Idly'

The black gram dhal was soaked into water for 8 hrs and was grinded in grinder. It was mixed with ravae (1:1 ratio) and was fermented for overnight. 'Idly' was prepared from mix in a 'idly’ mould by steaming for 15 min.

\section{'Sampar'}

The (dubai) drumstrick, yellow dhal, carrot, brinjal and tomatoes were washed well in water and were cooked in water with onions and chilly for $20 \mathrm{~min}$. Then coconut milk powder and chili powder were added and cooked well.

\section{'Sambol'}

The onions, chilly, salt were added and ground in a domestic grinder. Then scraped coconut was added and ground for $10 \mathrm{~min}$.

The ripped 'itharai' plantain was purchased in the local market.

\section{Analysis of foods}

All foods were analyzed for their total sugar (23), total protein (23), moisture (23), and soluble dietary fiber (24), insoluble dietary fiber (24), and total dietary fiber (24) contents.

\section{Subjects}

A group of 20 healthy volunteers (11 Males and 9 Females) between 20 to 22 years old was selected and the weight and height were determined and body mass index were calculated. The volunteers who had abnormal glucose tolerance, underweight or overweight, dieting or restricting their carbohydrate intake, suffering from any illness or food allergy were excluded from the studies.

\section{Ethical clearance}

Ethical clearance for this study was obtained from the 'Ethical Review Committee', Faculty of Medicine, University of Jaffna.

\section{Estimation of blood glucose level of volunteers}

The blood samples were collected and measured using semiautomated biochemical analyzer (TC 3300) by 3, 5-dinitrosalicylic acid (DNSA) method.

\section{Calculation of glycaemic response and glycaemic index values}

Glycaemic index and glycaemic response were calculated (25).

\section{Statistical analysis}

Glycaemic response and glycaemic index values of different types of bakery products were analyzed by Randomized Complete Block Design (RCBD) using SAS analytical package.

\section{Results}

Mean age and body mass index of the volunteers were $20.10( \pm 0.72)$ years, and $21.22( \pm 3.49) \mathrm{kgm}^{-2}$ respectively.

The glucose (75.0g) was orally administered to ten volunteers after overnight fasting; the blood glucose level was measured at 30, 60, 90 and $120 \mathrm{~min}$. The mean fasting blood glucose level was $90.80( \pm 8.70) \mathrm{mgdL}^{-1}$ and the mean blood glucose level at 30, 60, 90 and 120 min was 141.40 $( \pm 10.04), 122.10( \pm 6.15), 111.30( \pm 11.88)$ and $92.30( \pm 10.30)$ mgdL $^{-1}$ respectively (Table 2 ). The mean glycaemic response for glucose at 30, 60, 90 and 120 min was 50.60 ( \pm 10.30$), 31.30$ ( \pm 10.40$), 20.50( \pm 15.83)$ and $1.50( \pm 7.26)$ mgdL-1 respectively (Table 2 ). The blood glucose level reached peak at $30 \mathrm{~min}$ after administration of pure glucose. The glycaemic indexes of test foods were calculated by taking glycaemic response value to the glucose obtained at $30 \mathrm{~min}$.

The proximate compositions of 'idly, 'sambol', 'sampar' and 'itharai' plantain are shown Table 4.1. After overnight fasting (12 h), 75g digestible carbohydrate containing different combinations of selected black gram based combined food items were administered to the same volunteers on separate days. Peak glycaemic response to all the foods was obtained at $30 \mathrm{~min}$.

'Idly' (426.86g) with 'sambol' (25g) was administered to the volunteers, the mean fasting blood glucose level was $92.60( \pm 6.04) \mathrm{mgdL}^{-1}$ and the mean blood glucose levels at 30, 60, 90 and 120 min were 121.40 ( \pm 9.66$), 108.30$ $( \pm 11.77), 102.50( \pm 3.33)$ and $93.70( \pm 8.09) \mathrm{mgdL}^{-1}$ respectively (Table 2$)$. The mean change in glycaemic response at 30, 60, 90 and $120 \mathrm{~min}$ were 28.80 ( \pm 6.76$), 15.70$ ( \pm 9.43$), 9.90( \pm 7.05)$ and $1.10( \pm 4.07) \mathrm{mgdL}^{-1}$ respectively (Table 2). The mean GI value of 'idly' with 'sambol' was $56.85( \pm 6.26) \%$. 

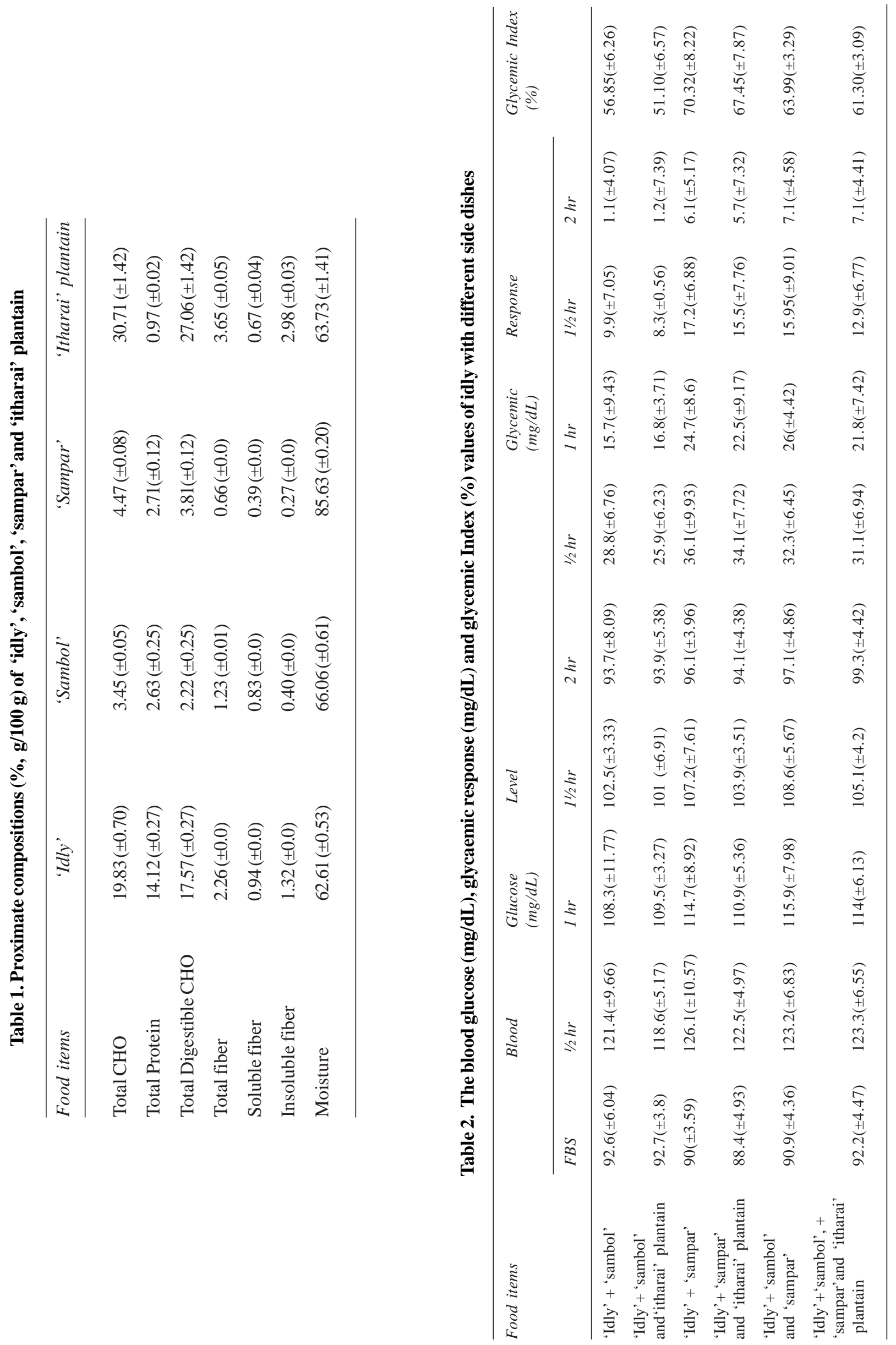
'Idly' (426.86g) with 'sambol' (25g) and 'itharai' plantain (50g) was administered to the volunteers, the mean fasting blood glucose level was $92.70( \pm 3.80) \mathrm{mgdL}^{-1}$ and the mean blood glucose levels at 30, 60, 90 and $120 \mathrm{~min}$ were $118.60( \pm 5.17), 109.50( \pm 3.27), 101.00( \pm 6.91)$ and $93.90( \pm 5.38) \mathrm{mgdL}^{-1}$ respectively (Table 4.2 ). The mean change in glycaemic response at 30,60, 90 and $120 \mathrm{~min}$ were 25.90 ( \pm 6.23$), 16.80$ ( \pm 3.71$), 8.30( \pm 0.56), 1.20$ ( \pm 7.39$)$ mgdL-1 respectively (Table 4.2 ). The mean GI value of 'idly' with 'sambol' and 'itharai' plantain was 51.10 $( \pm 6.57) \%$.

'Idly' (426.86g) with 'sampar' (25g) was administered to the volunteers, the mean fasting blood glucose level was $90.00( \pm 3.59) \mathrm{mgdL}^{-1}$ and the mean blood glucose levels at 30, 60, 90 and 120 min were $126.10( \pm 10.57), 114.70$ ( \pm 8.92$), 107.20( \pm 7.61)$ and $96.10( \pm 3.96)$ mgdL $^{-1}$ respectively (Table 4.2). The mean change in glycaemic response at 30, $60,90$ and $120 \mathrm{~min}$ were 36.10 ( \pm 9.93$), 24.70( \pm 8.60), 17.20$ ( \pm 6.88$)$ and $6.10( \pm 5.17)$ mgdL $^{-1}$ respectively (Table 4.2) The mean GI value of 'idly' with 'sampar' was 70.32 $( \pm 8.22) \%$.

'Idly' (426.86g) with 'sampar' (25g) and 'itharai' plantain (50g) was administered to the volunteers, the mean fasting blood glucose level was $88.40( \pm 4.93) \mathrm{mgdL}^{-1}$ and the mean blood glucose levels at 30, 60, 90 and $120 \mathrm{~min}$ were $122.50( \pm 4.97), 110.90( \pm 5.36), 103.90( \pm 3.51)$ and $94.10( \pm 4.38) \mathrm{mgdL}^{-1}$ respectively (Table 4.2$)$. The mean change in glycaemic response at 30, 60, 90 and $120 \mathrm{~min}$ were $34.10( \pm 7.72), 22.50( \pm 9.17), 15.50( \pm 7.76)$ and 5.70 $( \pm 7.32) \mathrm{mgdL}^{-1}$ respectively (Table 2 ). The mean GI value of 'idly' with 'sampar' and 'itharai' plantain was 67.45 $( \pm 7.87) \%$.

'Idly' (426.86g) with 'sambol' (25g) and 'sampar' (25g) was administered to the volunteers, the mean fasting blood glucose level was $90.90( \pm 4.36) \mathrm{mgdL}^{-1}$ and the mean blood glucose levels at 30, 60, 90 and 120 min were 123.20 ( \pm 6.83$), 115.90( \pm 7.98), 108.67( \pm 5.67)$ and $97.10( \pm 4.86)$ $\mathrm{mgdL}^{-1}$ respectively (Table 4.2 ). The mean change in glycaemic response at 30, 60, 90 and 120 min were 32.30 ( \pm 6.45$), 26.00( \pm 4.42), 15.95( \pm 9.01)$ and $7.10( \pm 4.58)$ $\mathrm{mgdL}^{-1}$ respectively (Table 2). The mean GI value of 'idly' with ‘sambol’ and ‘sampar’ was $63.99( \pm 3.29) \%$.

'Idly’ (426.86g) with ‘sambol’ (25g), 'sampar' (25g) and 'itharai' plantain (50g) was administered to the volunteers, the mean fasting blood glucose level was 92.20 $( \pm 4.47) \mathrm{mgdL}^{-1}$ and the mean blood glucose levels at 30, 60,90 and 120 min were $123.30( \pm 6.55), 114.00( \pm 6.13) 105.10$ $( \pm 4.20)$ and $99.30( \pm 4.42) \mathrm{mgdL}^{-1}$ respectively (Table 4.2). The mean change in glycaemic response at 30, 60, 90 and 120 min were 31.10 ( \pm 6.94$), 21.80( \pm 7.42), 12.90( \pm 6.77)$ and 7.10 ( \pm 4.41$) \mathrm{mgdL}^{-1}$ respectively (Table 2 ). The mean GI value of 'idly' with 'ambol', 'sampar' and 'itharai' plantain was $6 i .30( \pm 3.09) \%$.
The study indicated that the GI value of 'Idly' with 'sambol' or 'sambol' and 'itharai' plantain or 'sampar', 'sampar' and 'itharai' plantain or 'sambol' and 'sampar' or 'sambol', 'sampar' and 'itharai' plantain were 56.85 ( \pm 6.26$)$, 51.10 ( \pm 6.57$), 70.32( \pm 8.22), 67.45( \pm 7.87), 63.99( \pm 3.29)$ and $61.30( \pm 3.09) \%$ respectively (Table 4.2$)$.

The glycaemic response of glucose differed significantly $(\mathrm{p}<0.05)$ from 'idly' with 'sambol' or 'sambol' and 'itharai' plantain or 'sampar' and 'itharai' plantain or 'sambol' and 'sampar' or 'sambol', 'sampar' and 'itharai' plantain .The glycaemic response and glycaemic index values of 'idly' with 'sambol' differed significantly $(\mathrm{p}<0.05)$ from that of 'idly' with 'sambol' and 'itharai' plantain. The glycaemic response of 'idly' with 'sampar' did not differ significantly ( $p>0.05)$ from that of 'idly' with 'sampar' and pitharai' plantain, while the glycaemic index value of 'dly' with 'sampar' differed significantly $(\mathrm{p}<0.05)$ from 'idly' with 'sampar' and 'itharai' plantain. The glycaemic response and glycaemic index values of 'idly' with 'sambol' and 'sampar' did not differ significantly ( $p>0.05$ ) from 'idly' with 'sambol', 'sampar' and 'itharai' plantain.

\section{Discussion}

This was the first study indicated the GI value of 'Idly'based meals in Sri Lanka (Table 2). Based on these GI values, 'idly' with 'sambol' and plantain ('itharai') is of lower GI value (<55\%). 'idly’ either with ‘sambol' or 'sampar' and plantain ('itharai') or 'sambol' and 'sampar' or 'sambol', 'sampar' and plantain ('itharai') are of medium GI value (55 to 70\%). 'Idly’ with ‘sampar’ alone is high GI value (>70\%).

The mean glycaemic index value of 'idly' with 'sambol' [56.85 ( \pm 6.26$) \%$ ] was closer to that of 'idly' [60 ( \pm 2.0$) \%]$ (Parboiled and raw rice, black bhal, soaked, ground, fermented and steamed) with 'chutney' from a study in India (26) and much lower than that of 'idly' [77.0 ( \pm 2.0$)$ \%] with 'chutney' from another study in India (26). However the mean glycaemic index value of 'idly' with 'sambol' and 'sampar' and 'sambol', 'sampar' and 'itharai' plantain was closer to that of 'idly' with 'chutney' from India (26). Many people have raised concerns about the variation in published GI values for apparently similar foods. This variation may reflect both methodological factors and true differences in the physical and chemical characteristics of the foods. One possibility is that two similar foods may have different ingredients or may have been processed with a different method, resulting in significant differences in the rate of carbohydrate digestion and hence the GI value (26). Another reason GI values for apparently similar foods vary is that different testing methods are used in different parts of the world. Differences in testing methods include the use of different types of blood samples (capillary or venous), different experimental time periods, and different portions of foods ( $50 \mathrm{~g}$ of total rather than of available carbohydrate). 
Although capillary and venous blood glucose values have been shown to be highly correlated, it appears that capillary blood samples may be preferable to venous blood samples for reliable GI testing. After the consumption of food, glucose concentrations change to a greater degree in capillary blood samples than in venous blood samples. Therefore, capillary blood may be a more relevant indicator of the physiologic consequences of high-GI foods (26). In addition, it must be remembered that the GI values listed in the table for commercially available processed foods may change over time if food manufacturers make changes in the ingredients or processing methods used (26). This difference in the accuracy of measurements of the carbohydrate content might explain some of the variation in reported GI values for foods. Food labels may or may not include the dietary fiber content of the food in the total carbohydrate value, leading to confusion that can markedly affect GI values, especially those for high-fiber foods. Consequently, researchers should obtain accurate laboratory measurements of the available carbohydrate content of foods as an essential preliminary step in GI testing. The available carbohydrate portion of test and reference foods should not include resistant starch, but, in practice, this can be difficult to ensure because resistant starch is difficult to measure. There is also difficulty in determining the degree of availability of novel carbohydrates, such as sugar alcohols, which are incompletely absorbed at relatively high doses (26). Measuring the rate at which carbohydrates in foods are digested in vitro has been suggested as a cheaper and less time-consuming method for predicting the GI values of foods. However, only a few foods have been subjected to both in vitro and in vivo testing, and it is not yet known whether the in vitro method is a reliable indication of the in vivo postprandial glycemic effects of all types of foods. It is possible that some factors that significantly affect glycemia in vivo, such as the rate of gastric emptying, will not change the rate of carbohydrate digestion in vitro. For example, high osmolality and high acidity or soluble fiber slow down the gastric emptying rate and reduce glycemia in vivo, but they may not alter the rate of carbohydrate digestion in vitro (26). It is difficult to mimic all of the human digestive processes in a test tube. In fact, research results from our laboratory have shown that GI values measured in vivo can be significantly different for the same foods measured in vitro.

Among the different combinations of 'idly' studied with six different side dishes, 'idly' with 'sambol' and plantain is the best choice. Consumption of 'idly' either with 'sambol' or 'sambol' and plantain for those who need a low GI diet is therefore recommended. Although the dietary glycemic index is directly relevant in metabolic studies in which the total carbohydrate content is held constant, in free-living populations the amount of carbohydrate (e.g., as a percentage of energy) and its composition varies among individuals. Because the glucose and insulin responses depend on both the quantity and quality of the carbohydrate, we have used the dietary glycemic load, i.e., the amount of carbohydrate multiplied by its glycemic index, to represent both of these dimensions of carbohydrate intake. For an individual food, it is intuitively obvious that the glycemic load will be more relevant than the glycemic index. For calculating the total dietary glycemic load, the glycemic load scores from all foods are added. From a statistical perspective, the glycemic load also presents an interaction, as does any cross-product. This is physiologically relevant and makes sense intuitively because this interaction implies that the glycemic index is more important when the total carbohydrate content of the diet is high. Even though the above diets are selected for the diabetic and coronary heart disease patients, recommendation of the diets should be made after analyzing not only their GI, but also glycaemic load and energy contents should be considered.

The composition of the food or the meal will influence the blood glucose response. Further foods within the same classification can have different glycaemic indices. For example, the different varieties of potatoes, cereals showed variations in glycaemic indices $(27,28)$. Furthermore processing of the food influence the GI. During cooking gelatinization of starch takes place, the cell walls are ruptured and the starch molecules are released (29). Therefore structural integrity of cell wall and starch granules also determine the glycaemic index. However, the methods of preparations of 'sambol' and 'sampar' were different. The moisture contents of the both preparations varied significantly. Thus the GI of 'idly' with sampar was higher than that of 'sambol'. When the moisture content of the food is high the digestion of the food will be easier and hence the glucose release into the blood will be quicker (28). The moisture content of with sampar was higher than that of 'sambol' (Table 2).

When the total dietary fibre, soluble dietary fibre and insoluble dietary fibre contents are considered in the different basic diet in combination with different side dishes, no direct correlations with the changes in the GI were observed. An increase in the total fibre content of food can delay the glycaemic response (30). Fibre delays the digestion of starch in the stomach, transition time of the stomach contents to the duodenum, delay the diffusion of different saccharides in the duodenum, delay the hydrolysis of polysaccharides in the duodenum and delay the absorption of monosaccharides through the microvillai of the epithelial cells of the jejunum and the upper part of the ileum (31). Total dietary fibre content has a significant negative correlation with GI (32). Based on the results, the 'idly' with 'itharai plantain' was the best basic food having lowest GI with all different combinations of side dishes. With 'itharai plantain', the low GI was obtained with all different combinations of side dishes. When consumed without 'itharai plantain', the GI of all different combinations of side dishes increased (Table 2). The results from this study indicated that the GI of the 
diet does not depend only on the type of the basic food but also on the side dishes consumed. From the results it can be concluded that glycaemic index of a mixed diet is influenced by the ingredients included in the curries. Digestibility of sorgum, winged bean and horse gram was not influenced when it was supplemented with the red chilli, cumin, black pepper, coriander, garlic, asafoetide, dry ginger and ajowan (33). The digestibility of proteins of different legumes was decreased by chilli and coriander (34). Therefore it is not possible to support the increase in glycaemic index of the food combinations with gravy, due to added spices. Inclusion of gravy to the different basic foods increased the GI. This may be due to the increased digestibility with high moisture content of the foods. However, further research on this observation is necessary.

\section{Conclusion}

Based on these GI values, 'idly' with 'sambol' and plantain ('itharai') is of lower GI value (<55\%). 'Idly' either with 'sambol' or 'sampar' and plantain ('itharai') or 'sambol' and 'sampar' or 'sambol', 'sampar' and plantain ('itharai') are of medium GI value (55 to 70\%). 'Idly' with 'sampar' alone is high GI value (>70\%). When plantain ('itharai') was given with 'idly', the GI values were decreased, while mixed with 'sampar', the GI values were increased. The GI values were increased when the foods were consumed with 'sampar' alone or 'sampar' and 'sambol'.

\section{Recommendation}

Therefore, when providing dietary advice to diabetic subjects and those at risk of cardiovascular disease emphasis must be made on the importance of the side dish to a basic food item, prior be consumed. Consumption of food containing fiber diet will significantly reduce the rise in blood sugar level. However recommendation of the food should be made after analyzing the glycaemic index, glycemic load and energy contents of the food.

\section{Acknowledgement}

Financial assistance by Diagnostic Vision, George Steuart Agencies (Pvt) Ltd.

\section{References}

1. Chlup R, Bartek J, Reznickova M, Zapletalova J, Doubravova B, Chlupova L, Seckar P, Dovorackova S, Simanek V. Determination of glycaemic index of selected foods (white bread and cereal bars) in healthy persons. Biomed Papers 2004; 148(1): 17-25.

2. Anderson GH. Sugars and health. Nutrition Research 1997; 17(9): 1485-98.

3. Wolver TMS. Dietary recommendations for diabetes: high carbohydrate or high monounsaturated fat? Nutrition Today 1999; 34(2): 73-7.
4. Mendosa D. (2007). Glycaemic Index. Available from: http:/ /www.mendosa.com/gi:htm

5. Vrolix R, Van Meijl LEC, Mensink RP. The metabolic syndrome in relation with the glycaemic index and the glycaemic load. Physiology Behaviour 2008; 90: 293-9.

6. Augustin LS, Franceschi S, Jenkins DJA, Kendall CWC, Vecchia CL. Glycaemic index in chronic disease. European Journal of Clinical Nutrition 2002; 56: 1049-71.

7. Retterstol K, Hennig CB, Jversen PO. Improved plasma lipids and body weight in overweight / obese patients with type III hyperlipoproteinemia after 4 weeks on a low glycaemic diet. Clinical Nutrition 2009; 28: 213-5.

8. Ebbeling CB, Leidig MM, Feldman HA, Lovesky MM, Ludwig DS. Effects of low glycaemic load vs. low-fat diets in obese young adults. A randomized trial. Journal of American Medical Association 2007; 297(19): 2092-102.

9. Amano V, Sugiyama M, Lee JS, Kawakubo KK, Mori K, Tang AC, Akabayashi A. Gylcemic index-based nutritional education improves blood glucose control in Japanese adults. Diabetes Care 2007; 30(7): 1874-6.

10. Abete I, Parr D, Martinez JA. Energy-restricted diets based on a distinct food selection affecting the glycaemic index induce different weight loss and oxidative response. Clinical Nutrition 2008; 27: 545-51.

11. Jenkins DJA, Kendall CWC, Augustin LSA, Franceschi S, Hamidi M, Marchie A, Jenkins AL, Axelsen M. Glycaemic index: Overview of implications in health and disease. American Journal of Clinical Nutrition 2002; 76 (Suppl): 266S-273S.

12. Brand-Miller JC, Holt SHA, Powlak DP, McMillan J. Glycaemic index and obesity. American Journal of Clinical Nutrition 2002; 76 (Suppl.): 281S-285S.

13. Burani J, Lango PJ. Low Glycaemic Index carbohydrates. An effective behavioral change for glycaemic control and weight management in patients with Type 1 and 2 diabetes. The Diabetes Educator 2006; 32(1): 78-88.

14. Sheard NF, Clark NG, Brand-Miller JC, Franz MJ, Pi-Sunyer FX, Mayer-Davis E, Kulkarni K, Geil P. Dietary carbohydrate (amount and Type) in the prevention and management of diabetes: A statement by the American Diabetes Association. Diabetes Care 2004; 27(9): 2266-71.

15. Villegan R, Liu S, Gao YT, Yang G, Li H, Zheng,W, Shu XO. Prospective study of diatery carbohydrates glycaemic index, glycaemic load and incidence of type 2 diabetes mellitus in middle-aged Chinese women. Achieves International Medicine 2007; 167(21): 2310-16.

16. Sone H, Yoshimura Y, Tanaka S, Iimuro S, Ohashi Y, Ito H, Seino H, Ishibashi S, Akamuna Y, Yamada N. (Crosssectional association between BMI, glycaemic control and energy intake in Japanese patients with type 2 diabetes. Analysis from the Japan diabetes complication study. Diabetes Research Clinical Practise 2007; 775: S23-29. 
17. Beulens JWJ, de Bruijne LM, Stolk RP, Peeters PHM, Bots ML, Grobbee DG, Vander Schouw, YT. High dietary glycaemic load and glycaemic index increases risk of cardio vascular disease among middle - aged women. Journal of American College of Cardiology 2007; 50(1): 14-21.

18. Berra B, Rizzo AM. Glycaemic index, glycaemic load, wellness and beauty: the state of the art. Clinical Dermatology 2009; 27: 230-5.

19. Mursu J, Virtanen JK, Rissanen TH, Tuomainen TP, Nykanen I, Laukkanen JA, Kortelainen R, Vaulilainen S. Glycaemic index, glycaemic load, and the risk of acute myocardial infarction in Finnish men. The Kuopio ischaemic heart disease risk factor study. Nutrition Metabolism Cardiovascular Disease 2009; 20: 1-6.

20. Murakami K, Sasaki S, Takahashi Y, Uenishi K, Watanabe T, Kohri T, Yamasaki M, Watanabe R, Baba K, Shibata K, Takahashi T, Hayabuchi, H, Ohki K, Suzuki, JO. Dietary glycaemic index is associated in the decreased premenstrual symptoms in young Japanese women. Nutrition 2008; 24: 554-61.

21. Murakami K, Miyake Y, Sasaki S, Tanaka K, Fukushime W, Kiyohara C, Tsuboi Y, Yamada T, Oeda T, Miki T, Kawanura N, Sakae N, Fukugama H, Hirota Y, Nagai M. Dietary glycaemic index is inversely associated with the risk of Parkinsons disease: A case control study in Japan. Nutrition 2009 25: 1-7.

22. Valtuena S, Pellegrini N, Ardigo D, Rio DD, Numeroso F, Scazzina F, Monti L, Zavaroni I, Brighenti, F. Dietary glycaemic index and liver steatosis. American Journal of Clinical Nutrition 2006; 84: 136-42.

23. Pearson D, eds. The Chemical analysis of foods. 7th ed. Edinburgh, London, Churchill. Livingstone (1976).

24. Megazyme, Total dietary fibre assay procedure. Aviable from: http://www.megazyme.com/booklets/KTDFR.pdf, 2007.
25. Wolever TMS, Jenkins DJA, Jenkins AL, Josse RG. The Glycemic Index. Methodology and Clinical Implications. American Journal of Clinical Nutrition 1991; 54: 846-54.

26. Foster-Powell K, Holt SHA, Brand miller JC. International table of glycaemic index and glycaemic load values. The American Journal of Clinical Nutrition 2002; 76: 5-56.

27. Thathvasuthan A, Chandrasekara DG, Wijesinghe NG, Jayawardena HMDK. The effect of types of cereal on the glycaemic response of two traditional Sri Lankan food items. Tropical Agriculture Research 2007; 19: 101-19.

28. Fernandes G, Velangi A, Wolewer TMS. Glycaemic index of potatoes commonly consumed in North America. Journal of the American Diebetic Association 2005; 105: 557-62.

29. Wandsnider LA. The roasted and the boiled: food composition and heat treatment with special emphasis on Pil-Hearth cooking. Journal of Anthropological Archaeology 1997; 16: 1-48.

30. Marangoni F, Poli A. The glycaemic index of bread and biscuits is markedly reduced by the addition of a proprietary fiber mixture to the ingredients. Nutrition, Metabolism and Cardiovascular Diseases 2008; 18: 602-5.

31. Nishimune T, Yalcushiji T, Summoto T. Glycaemic response and fiber content of some foods. American Journal of Clinical Nutrition 1991 54: 414-19.

32. Hettiarachchi UPK, Ekanyae S, Welihinda J. Do Sri Lankan meals help decrease blood glucose response? Ceylon Medical Journal 2009; 54: 39-43.

33. Pradeep KU, Geervani P, Eggum BO. Influence of spices on utilization of sorgum and chickpea protein. Plants Foods and Human Nutrition 1991; 41: 269-76.

34. Prakash VHP, Prakash J. In vitro proteins digestibility of legumes cooked with spices. Food 1999; 43: 19-21. 